\title{
Un tératome du plancher buccal
}

\author{
Mohammed Moumine ${ }^{1, *}$, Faical Choumi ${ }^{1}$, Hicham Sabani ${ }^{2}$, Mohammed Karim El Khatib ${ }^{2}$, Mohammed Naasih ${ }^{1}$ \\ Service de stomatologie et chirurgie maxillo-faciale, hôpital militaire Moulay Ismail, Meknès, Maroc \\ Service de stomatologie, chirurgie maxillo-faciale et chirurgie plastique, hôpital militaire d'instruction Mohammed V, Rabat, Maroc \\ mmoumine@yahoo.com
}

(Reçu le 15 avril 2014, accepté le 18 septembre 2014)

Mots clés : tératome / plancher buccal /
choristome

Key words:

teratoma / floor of the mouth / choristoma
Résumé - Introduction : Les tératomes du plancher buccal sont des choristomes rares, de découverte exceptionnelle chez l'adulte. Observation : Un cas rare de tératome du plancher buccal chez un patient de 41 ans est rapporté. Il se manifestait par une tuméfaction du plancher buccal, mobilisable à la palpation. L'examen radiographique et la tomodensitométrie (TDM) révélaient une image ostéocondensante incluse dans une masse graisseuse. L'examen anatomopathologique de la pièce d'exérèse concluait à un tératome. Discussion : Le diagnostic de tératome doit être évoqué devant une radio-opacité du plancher buccal. L'orthopantomogramme, la TDM et ou l'imagerie par résonance magnétique (IRM) orientent le diagnostic. Le diagnostic de certitude est anatomopathologique.

Abstract - A teratoma of the lateral floor of the mouth. Introduction: Teratomas of the floor of the mouth are uncommon choristomas. Discovery of teratoma in adults is rare. Observation: An exceptional case of a teratoma of the floor of the mouth in a 41-year-old patient is reported. It was manifested by swelling of the floor of the mouth and could be mobilized by palpation. Radiographic examination and a CT scan revealed a dense mineralized image included in a fat mass. The histopathological analysis concluded it was a teratoma. Comments: The diagnosis of teratoma should be considered for radio-opacity of the floor of the mouth. An orthopantomogram, computed tomography (CT) and/or Magnetic Resonance Imaging (MRI) guide the diagnosis. The diagnosis is histopathological.

\section{Observation}

Un patient âgé de 41 ans, sans antécédent particulier, avait consulté il y a un an pour une tuméfaction du plancher buccal en regard du secteur molaire de la face interne de la branche horizontale gauche de la mandibule. L'apparition des symptômes datait de trois mois. À l'admission, l'examen exobuccal avait trouvé une tuméfaction au niveau sous-angulo-mandibulaire gauche, mobile par rapport à la peau à la palpation bidigitale. L'examen endobuccal avait révélé une tuméfaction souple indolore fluctuante d'environ $4 \mathrm{~cm}$ sur $2 \mathrm{~cm}$, mobile par rapport à la mandibule non adhérente à la muqueuse. Il n'existait pas d'adénopathie ni de signe salivaire ou neurologique, notamment pas de retentissement sur le nerf lingual. L'orthopantomogramme mettait en évidence une opacité semblable à une dent en regard de la racine mésiale de la 36 (Fig. 1a), et la TDM avec injection de produit de contraste montrait une masse graisseuse incluant des opacités ressemblant à deux dents situées en sus-mylohyoïdien (Fig. 1b) faisant suspecter un tératome. Le traitement avait consisté en une exérèse en monobloc par voie endobuccale sous anesthésie générale ; les suites opératoires et la cicatrisation ont été simples. L'examen anatomopathologique de la pièce opératoire mettait en évidence un tissu fibro-adipeux mature et osseux anarchique, compatible avec un tératome bénin.

\section{Discussion}

Les tératomes du plancher buccal sont des malformations congénitales rares. Ils font partie des kystes et fistules du premier arc branchial [1]. Le tératome fait partie des choristomes (du grec choristos : séparé ; ome : tumeur), dans le groupe des hétérotopies tissulaires [1]. Les tératomes congénitaux sont observés dans $1 / 4000$ naissances, avec une prédominance féminine. Leur localisation au niveau du plancher buccal constitue environ $1,6 \%$ à $6,5 \%$ de toutes les localisations [1] et leur découverte chez l'adulte reste exceptionnelle [2]. Les tératomes du plancher buccal sont souvent retrouvés en position médiane mais dans $6 \%$ des cas ils sont latéraux [35]. Ils sont observés presque exclusivement chez les enfants mais ils peuvent se manifester exceptionnellement chez les adultes durant les deuxième et troisième décennies de la vie avec une prédominance féminine [5]. Macroscopiquement, un tératome peut être kystique, solide ou mixte. Il comporte des tissus étrangers à la région qui l'abrite, ressemblant à 


\section{A S C L I N I Q U E}

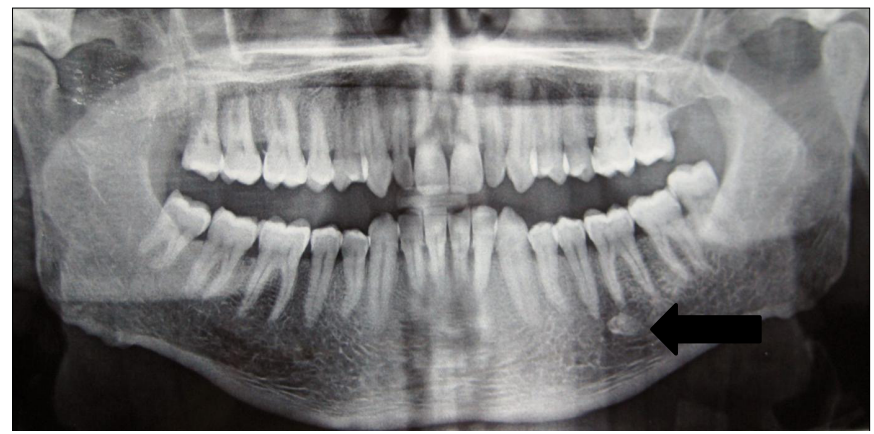

Fig. 1a. Orthopantomogramme montrant une opacité dentaire en regard de la racine mésiale de la 36.

Fig. 1a. Orthopantomogram shows a dental opacity under the mesial root of 36 .

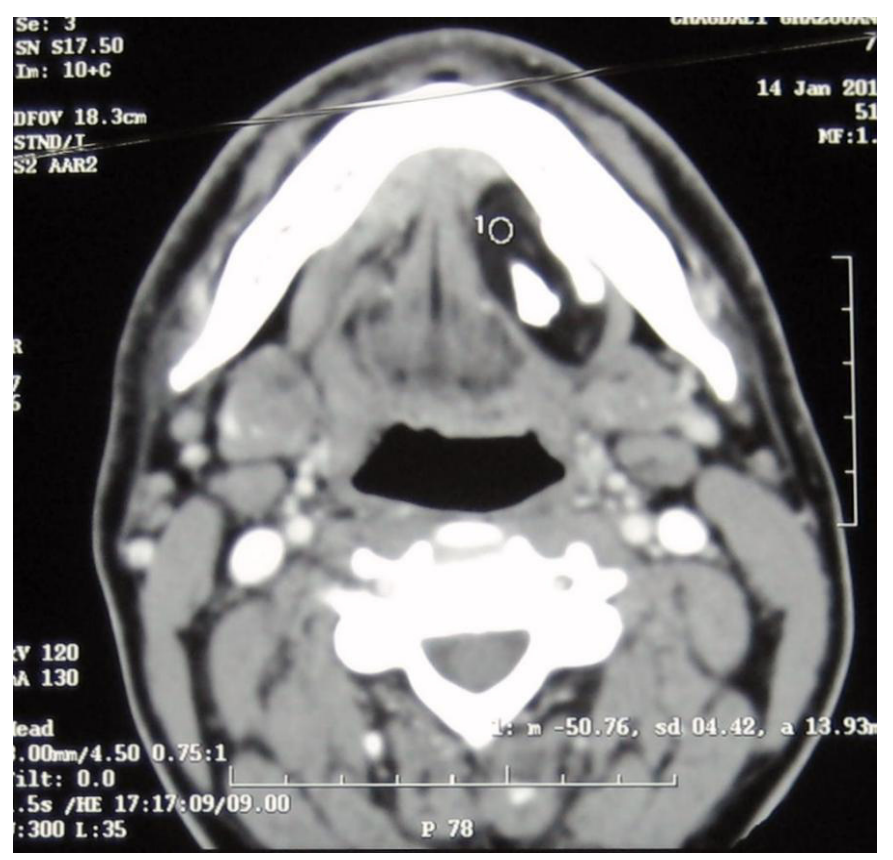

Fig. 1b. TDM avec injection de produit de contraste montrant une tumeur graisseuse incluant des opacités dentaires.

Fig. $1 b$. CT with injection of contrast medium showing a fatty tumor including dental opacities.

ceux qui se succèdent au cours du développement à partir des trois feuillets embryonnaires : ectoblaste, mésoblaste et endoblaste [1]. Le tissu neural est le plus fréquent [6]. L'orthopantomogramme et/ou la TDM avec injection de produit de contraste suspectent le diagnostic sur la présence d'une opacité similaire à une dent comme dans le cas rapporté. Le caractère hétérogène de la tuméfaction à l'IRM oriente le diagnostic et permet de planifier le traitement chirurgical. Le diagnostic de certitude est obtenu grâce à l'examen histologique de la pièce opératoire [4]. Macroscopiquement, le tératome malin est plus souvent solide que kystique et contient des tissus immatures. Le diagnostic est posé devant la présence de cellules immatures, mais le degré de malignité n'est pas proportionnel à la quantité de tissus immatures présents dans le tératome [1-8]. Meyer [7] avait proposé une classification anatomochirurgicale pour les tératomes du plancher buccal basée sur leur situation par rapport aux muscles génioglosse, géniohyoïdien, mylohyoïdien et digastrique. Le cas rapporté est classé sublingual. Le risque de cancérisation se voit essentiellement dans les tératomes des gonades, notamment les tératomes ovariens, et son incidence est liée à l'âge de découverte [2]. Pour les tératomes du plancher buccal, le risque de transformation maligne est exceptionnel [2]. Le traitement des tératomes est l'exérèse chirurgicale complète. Le choix de la voie d'abord se fait en fonction de la situation et de la taille de la tumeur. La voie endobuccale est indiquée lorsque la tumeur est sus-mylohyoïdienne, un abord cervical est préféré lorsque la tumeur est sous-mylo-hyoïdienne. La récidive est rare, généralement liée à une exérèse incomplète.

\section{Conflits d'intérêt : aucun}

\section{References}

1. Benouaiche L, Couly G, Michel B, Devauchelle B. Diagnostic et prise en charge des tératomes cervicofaciaux congénitaux : à propos de quatre cas, revue de la littérature et mise au point. Ann Chir Plast Esthet 2007;52:114-23.

2. Boko E, Amaglo K, Kpemissi E. A bulky dermoid cyst of the floor of the mouth. Eur Ann Otorhinolaryngol Head Neck Dis 2014;131:131-4.

3. Lin HW, Silver AL, Cunnane ME, Sadow PM, Kieff DA. Lateral dermoid cyst of the floor of mouth: unusual radiologic and pathologic findings. Auris Nasus Larynx 2011;38:650-3.

4. Ariyoshi $Y$, Shimahara M. Magnetic resonance imaging of a submental dermoid cyst: report of a case. Oral Maxillofacial Surg 2003;61:507-10.

5. King RC, Smith BR, Burk JL. Dermoid cyst in the floor of the mouth. Review of the literature and case reports. Oral Surg Oral Med Oral Pathol 1994;78:567-76.

6. Ferlito A, Devaney K0. Clinicopathological consultation developmental lesions of the head and the neck: terminology and biologic behaviour. Ann Oto Rhinol Laryngol 1995;104:913-8.

7. Meyer I. Dermoid cysts (dermoids) of the floor of the mouth. Oral Surg Oral Med Oral Pathol 1955;8:1149-64.

8. Azizkhan RG, Haase GM, Applebaum H, Dillon PW, Coran AG, King PA. Diagnosis, management, and outcome of cervicofacial teratomas in neonates: a Children's Cancer Group study. J Pediatr Surg 1995;30:312-6. 